\title{
Dieta de sarapós da família sternopygidae em ambientes de praia no Rio Negro, Amazonas
}

Os ambientes de praia na Amazônia Central ocorrem de forma aparente nas margens de rios, paranás e lagos, através da alternância do nível da água, que ocorre a partir do ciclo hidrológico, surgindo durante a fase estacional terrestre mais conhecida como os períodos de vazante e seca. Estes habitats proporcionam abrigo e fonte de alimentação para uma diversa e abundante ictiofauna, dentre as espécies de peixes que habitam os ambientes de praias destacam-se os peixes da ordem Gymnotiformes, os quais são conhecidos popularmente como sarapós ou peixes elétricos, sendo este último, devido a capacidade de gerar uma corrente de carga elétrica para eletrolocação e interações sociais, principalmente para a família Sternopygidae, utilizada na presente pesquisa. Estudos acerca dos hábitos alimentares e dieta dos Gymnotiformes são escassos e, essa falta de conhecimento tanto pela história de vida, comportamento e ecologia são os principais pontos para o não entendimento das pesquisas realizadas, sendo assim, este estudo é primordial para a melhor compreensão dos hábitos alimentares das espécies Eigenmannia macrops, Eigenmannia trilineata e Eigenmannia virescens coletadas em praias do rio Negro. Foram analisados ao total 180 estômagos, 60 de cada espécie e todos apresentaram algum item alimentar, estando os estômagos parcialmente cheios (0-25\%) até mesmo distendidos (75-100\%) como se destacou para os espécimes de $\mathrm{E}$. macrops. O item alimentar mais frequente foi o de inseto, seguido por material indefinido (digerido) e por fim o item material vegetal. A partir do IAi (\%) pode-se classificar as espécies $\mathrm{E}$. macrops $(>70 \%)$ e E. virescens $(>70 \%)$ na guilda trófica como insetívoras e E. trilineata como onívora com tendência a insetivoria. O valor calculado para amplitude de nicho trófico apresentou nível intermediário para E. trilineata e E. virescens e baixa para E. macrops, portanto tendo a maior dissimilaridade quanto aos itens consumidos. Quanto ao fator de condição, a espécie E. macrops demonstrou que estava em boas condições de bem-estar mesmo em período de seca, já as espécies E. trilineata e E. virescens apresentaram valores abaixo de zero, o que não as impossibilitou de explorar os recursos alimentares.

Palavras-chave: Praias; Alimentação; Sarapó; Dieta.

\section{Diet of sarapós of the family sternopygidae in beach environments in Rio Negro, Amazonas}

\begin{abstract}
The beach environments in Central Amazonia appear in an apparent way on the bench of rivers, paranás and lakes, through the alternation of the water level, which occurs from the The beach enver abundant ichthyy, apearing during the terrestral seasonal phase better known as the ebb and flow periods dry. These habitats provide shelter and a source of food for a diverse and abundant ichthyofauna. Among the species of fish that inhabit the beach environments, the fish of the order Gymnotiformes stand out, which are popularly known as sarapos or electric fish, the latter being, due to the ability to generate an electric charge current for electrolocation and social interactions, mainly for the family Sternopygidae, used in this research. Studies
about the Gymnotiformes' eating habits and diet are scarce and this lack of knowledge due to the history of life, behavior and ecology are the main points for the lack of understanding of the researches carried out, therefore, this study is essential for a better understanding of the feeding habits of the species Eigenmannia macrops, Eigenmannia trilineata and Eigenmannia virescens collected from Rio Negro beaches. A total of 180 stomachs were analyzed, 60 of each species and all presented some food item, with the stomachs being partially full (0-25\%) even distended $(75-100 \%)$, as was the case for E. macrops specimens. The most frequent food item was that of insects, followed by undefined (digested) material and finally the plan material item. From the IAi (\%), the species E. macrops (> 70\%) and E. virescens (>70\%) can be classified in the trophic guild as insectivores and E. trilineata as omnivores with a tendency to become insectivores. The calculated value for trophic niche amplitude species E. trilineata and E. virescens showed values below zero, which did not prevent them from exploring food resources.
\end{abstract}

Keywords: Beaches; Food; Sarapó; Diet.

Topic: Desenvolvimento, Sustentabilidade e Meio Ambiente

Reviewed anonymously in the process of blind peer.
Received: 04/02/2021

Approved: 25/02/2021
Wallacy Adriano Cavalcante Campos (iD) Universidade Federal do Amazonas, Brasil http://lattes.cnpq.br/8882652079165986 http://orcid.org/0000-0002-6640-3932

wallacyadrianoo@gmail.com

Thaynara Sofia Gomes Vieira (iD

Universidade Federal do Amazonas, Brasil http://lattes.cnpq.br/4523182495178618

http://orcid.org/0000-0002-0265-0731

thaysofia13@gmail.com

Kedma Yamamoto (iD

Universidade Federal do Amazonas, Brasil http://lattes.cnpq.br/0760543767899807

http://orcid.org/0000-0002-1026-2712

kcyamamoto@gmail.com
Referencing this:

CAMPOS, W. A. C.; VIEIRA, T. S. G.; YAMAMOTO, K. C.. Dieta de sarapós da família sternopygidae em ambientes de praia no Rio Negro, Amazonas. Revista lbero Americana de Ciências Ambientais, v.12, n.2, p.482-492, 2021. DOI: http://doi.org/10.6008/CBPC21796858.2021 .002 .0041 


\section{INTRODUÇÃO}

A região amazônica apresenta a maior bacia hidrográfica do mundo, possuindo cerca de 7,3 milhões de $\mathrm{km}^{2}$ de área, abrangendo aproximadamente 60\% do território nacional (JUNK et al., 1989), com mais de 2.700 espécies de peixes propriamente descritas para a bacia amazônica (REIS et al., 2016; DAGOSTA et al., 2019) e elevada heterogeneidade de habitats. Essa heterogeneidade é representada por ambientes como: igarapés, leito de grandes rios, bancos de vegetações flutuantes (macrófitas aquáticas), corredeiras, igapós, lagos e praias (LOWE-MCCONNEL, 1999). Os ambientes de praia na Amazônia Central ocorrem de forma aparente nas margens de rios, paranás e lagos, através da alternância do nível da água, que ocorre a partir do ciclo hidrológico, os quais surgem na fase estacional terrestre, mais conhecidas como períodos de vazante e seca (JUNK et al., 1989; ZUANON et al., 2008).

Durante a fase das águas baixas, as praias são ambientes que proporcionam abrigo para uma diversa e abundante icitiofauna (GOULDING, 1997), fonte de alimentação, inclusive para as que possuem o habito alimentar piscívoro, porém é baixa a disponibilidade de alimentos para peixes de pequeno porte. Segundo um autor, espécies que possuem na composição de sua dieta o consumo de pequenos invertebrados preferem praia aos ambientes de várzea. A alimentação dos peixes, segundo (SILVA, 2016), varia de acordo com a disponibilidade de alimento no ambiente, assim, apresentando para a grande maioria dos peixes uma plasticidade alimentar em sua dieta.

Dentre as espécies que habitam os ambientes de praias destacam-se os peixes da ordem Gymnotiformes que possuem o nome popular de sarapós, ou conhecidos como "peixes elétricos". A família Gymnotidae pertencente à ordem Gymnotiformes e, é compreendida pelos peixes neotropicais que possuem um sistema eletrogênico e eletrosensorial conjunto (QUEIROZ et al., 2013). O órgão elétrico e os eletrorreceptores dos Gymnotiformes conferem a estes animais a capacidade de produzir e detectar campos elétricos, sendo a mais conhecida especialização apresentada por esses peixes que permite aos mesmos usar sua própria geração de campo elétrico e eletrorrecepção não só para localização de objetos e alimentos, mas também para interações sociais como defesa territorial e comportamento sexual através de modulações das descargas dos órgãos elétricos (DOEs) (CRAMPTON et al., 2006). Os gymnotídeos abrangem dois gêneros, representados por 38 espécies, um pertencente ao gênero Electrophorus e 37 espécies ao Gymnotus (MILHOMEN et al., 2012).

Estudos sobre a biologia e hábitos alimentares dos Gymnotiformes são escassos. Gymnotiformes que habitam riachos amazônicos são fundamentais na ictiofauna porque atuam como ligações entre artrópodes detritívoros e outros organismos do meio aquático devido à sua dieta bentívora composta principalmente de presa autóctone como mencionado por (GIORA et al., 2014). No estudo apresentado por (INACIO et al., 2016), a espécie E. trilineata apresentou hábito de insetivoria, mais especificamente de insetos aquáticos, o que é representado por outros autores para peixes da família Sternopygidae, como no caso da Eigenmania virescens, que em sua alimentação apresentou larvas de Coleoptera e Chironomidade (SOARES, 1979), e para a dieta de Gymnotus cf carapo, cujo habito alimentar se adequa a região onde vive e que tem preferência por larvas de insetos, que são de fácil digestão (RESENDE, 2006). Estudos acerca dos 
hábitos alimentares e dieta dos Gymnotiformes são escassos e, essa falta de conhecimento tanto pela história de vida, comportamento e ecologia são os principais pontos para o não entendimento dos estudos realizados (GIORA, 2004). Diante disto, este trabalho é essencial para melhor compreensão dos hábitos alimentares das espécies Eigenmannia macrops, Eigenmannia trilineata e Eigenmannia virescens, gerando informações sobre a ecologia destes, e a interação com os ambientes de praia do médio Rio Negro.

\section{METODOLOGIA}

\section{Áreas de coleta}

As coletas das espécies de Eigenmannia macrops e Eigenmannia virescens ocorreram durante o período hidrológico de seca (águas baixas) em outubro/novembro de 2016, em ambientes de praia no baixo Rio Negro, divididas em duas localidades, sendo a primeira localizada no Parque Nacional de Anavilhanas (PARNA-Anavilhanas, $2^{\circ} 28^{\prime} 24,71^{\prime \prime} \mathrm{S}, 60^{\circ} 59^{\prime} 48,41^{\prime \prime} \mathrm{W}$ e $2^{\circ} 28^{\prime} 35,98^{\prime \prime} \mathrm{S}, 60^{\circ} 58^{\prime} 2,06^{\prime \prime} \mathrm{W}$ ), unidade de conservação federal situado no município de Novo Airão - Amazonas, e na segunda na Reserva de Desenvolvimento Sustentável Tupé (REDES - Tupé, $3^{\circ} 2^{\prime} 57,08^{\prime \prime} \mathrm{S}, 60^{\circ} 15^{\prime} 8,06^{\prime \prime} \mathrm{W}$ e $3^{\circ} 2^{\prime} 32,82^{\prime \prime} \mathrm{S}, 60^{\circ} 14^{\prime} 44,12^{\prime \prime}$ W), mais precisamente na praia do Tupé, no município de Manaus.

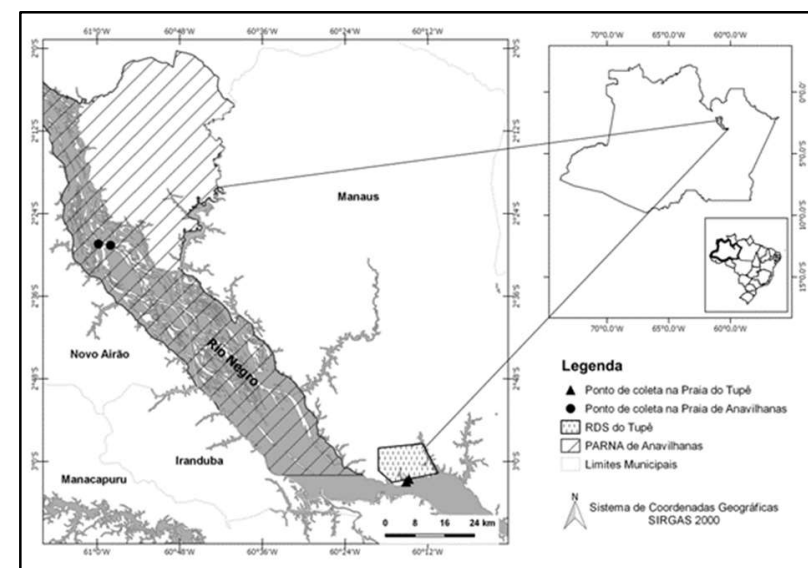

Figura 1: Localização das praias onde foram realizadas as amostragens no baixo Rio Negro.

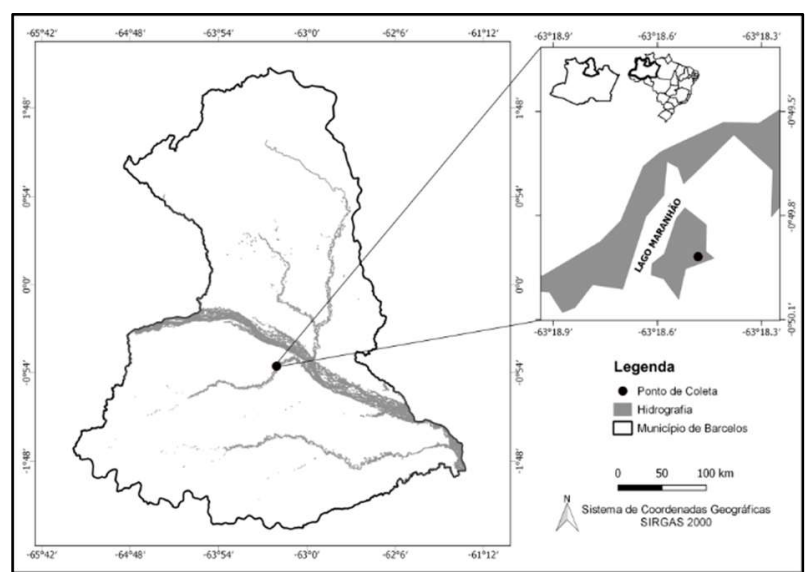

Figura 2: Localização onde foram realizadas as amostragens no município de Barcelos.

A coleta dos indivíduos da espécie de Eigenmannia trilineata ocorreu durante o período de seca, em novembro de 2005 , em praia do município de Barcelos $\left(00^{\circ} 49^{\prime} 55,12^{\prime \prime} \mathrm{S}\right.$ e $\left.63^{\circ} 18^{\prime} 28,95^{\prime \prime}\right)$, distante cerca de $490 \mathrm{~km}$ da capital Manaus.

\section{Amostragens}

Os pontos amostrais foram escolhidos aleatoriamente para cada ambiente de praia, estes, claro, considerando as possibilidades da realização dos arrastos. Determinou-se uma área mínima de $20 \mathrm{~m}$ de distância a contar da margem em direção perpendicular ao canal principal, livres da presença de substratos que impossibilitassem a coleta. Foram realizados 5 arrastos manuais consecutivos em cada ponto dos sítios amostrais, com rede de $20 \mathrm{~m}$ de comprimento $\times 2 \mathrm{~m}$ de altura e $5 \mathrm{~mm}$ entre nós opostos. Os arrastos ocorreram no período diurno de 07:00h às 10:00h e noturno de 19:00h às 22:00h. 
Os peixes coletados foram acondicionados em sacos plásticos, etiquetados com todas as informações do local, horário e data, após isto foram fixados em formalina a $10 \%$ de concentração e transportados para o laboratório de Ictiologia da Universidade Federal do Amazonas - UFAM, e acondicionados em frascos.

No laboratório, os peixes foram identificados através de chaves de identificação e de especialistas A biometria foi realizada coletando-se os dados de peso em gramas (g), com auxílio de balança de precisão (marca da balança) e o comprimento total (CT) em centímetros $(\mathrm{cm})$ no ictiômetro.

\section{Análise da composição alimentar e guildas}

A dieta foi determinada através da análise do conteúdo estomacal, a partir de observação em estereomicroscópio (Leica ES2), iniciando com a análise do Grau de Repleção Estomacal, que permite identificar a quantidade de alimento em cada estômago à olho nu ou com auxílio de microscópio para determinar os itens. Essa análise possui as seguintes classificações: Grau 0 = para estômagos vazios;

Grau I = para estômagos preenchidos até 25\%; Grau II = para estômagos preenchidos até 50\%; Grau III = para estômagos preenchidos até 75\%; Grau IV = para estômagos preenchidos 100\%

Em sequência foi obtida a frequência de ocorrência (F.O.), que consiste na somatória dos estômagos que possuem o mesmo item alimentar, dividindo pelo número de estômagos analisados (HYSLOP, 1980), calculada conforme a fórmula: F.O. = Li.100/ N, onde: F. O. = Frequência de ocorrência; $\mathrm{i}=$ Número de estômagos em que cada item alimentar foi identificado; $\mathrm{N}$ = Número de estômagos analisados com alimento.

O volume relativo também foi obtido, no qual é a estimativa visual do volume de cada item alimentar em relação ao volume total do alimento em cada estômago, em percentagem (HYSLOP, 1980).

Os resultados individuais dos métodos da frequência de ocorrência (F.O) e do volume relativos foram combinados no Índice Alimentar (IAi) (KAWAKAMI et al., 1980) e expressos em percentagem para cada item, adotando a expressão: $\mathrm{IAi}=\mathrm{Fi} * \mathrm{~V} / \Sigma\left(\mathrm{Fi}^{*} \mathrm{~V}\right)$ onde, $\mathrm{i}=$ item alimentar; $\mathrm{F}=$ frequência de ocorrência (\%) do determinado item; V= volume (\%) de determinado item. Este índice avalia o grau de importância que cada alimento possuiu na dieta dos peixes.

O item alimentar inseto foi identificado a partir de chave taxonômica (HAMADA et al., 2012) e ajuda de especialistas. A classificação das espécies em guildas tróficas foi realizada de acordo com a categoria alimentar e os valores de Índice Alimentar (IAi\%) correspondente a $\geq 70 \%$ (MÉRONA et al., 2008).

Para corroborar os resultados da composição alimentar e do índice alimentar, foi calculada a amplitude do nicho trófico utilizando os dados de volume através do índice padronizado de Levins, dado pela fórmula: $B A=[(\Sigma j P 2 i j)-1-1](n-1)-1$ onde: $B A=$ Amplitude do nicho trófico padronizada; $P i j=$ proporção do item alimentar $j$ na dieta da espécie $i ; \mathrm{n}=$ número total de itens alimentares.

Este índice varia de 0 a 1, ou seja, quanto mais próximo à 1 significa que os indivíduos consomem igualmente os recursos disponíveis e quanto mais próximo à 0 , maior é a dissimilaridade quanto aos itens consumidos. Além disso, a amplitude de nicho pode ser considerada baixa $(0-0,39)$, intermediária $(0,4-0,6)$ 
ou alta (0,61-1) (CORRÊA et al., 2011).

O fator de condição proposto por Fulton foi calculado segundo a fórmula: KF = (W / L3) x 100 onde: $\mathrm{KF}=$ Fator de condição de Fulton; $\mathrm{W}=$ Peso corporal em gramas; $\mathrm{L}=$ Comprimento corporal total em centímetros; 3 = coeficiente da regressão.

O fator de condição de Fulton assume que o crescimento é isométrico $(b=3,0)$, sendo determinado pela relação (peso/comprimento corporal). O fator de condição fornece importantes informações sobre o estado fisiológico do animal.

\section{RESULTADOS}

\section{Composição alimentar}

Foram analisados 180 indivíduos, distribuídos em 60 da espécie de Eigenmannia macrops, 60 da espécie Eigenmannia trilineata e 60 de Eigenmannia virescens. Da espécie E. macrops 28 indivíduos foram advindos do Parque Nacional de Anavilhanas e 32 da RDS Tupé; E. trilineata 60 advindos Barcelos; $E$ virescens 60 indivíduos do Parque Nacional de Anavilhanas.

A partir do grau de repleção obtido nas análises, todos os 180 estômagos apresentaram algum alimento. Eigenmannia macrops mostrou 7 estômagos na escala de 25\%, 9 na escala de 50\%, 16 com 75\% e 28 estômagos com 100\%. Já Eigenmannia trilineata apresentou 32 estômagos com 25\%, 21 com 50\%, 7 com 75\% e nenhum estômago apresentou 100\% de grau de repleção; Eigenmannia virescens mostrou 11 estômagos com 25\%, 19 com 25\% 22 com $75 \%$ e 8 com 100\% na escala do grau de repleção.

Tabela 1: Grau de repleção encontrado para os estômagos das espécies utilizadas no estudo.

\begin{tabular}{lllllll}
\hline & Eigenmannia macrops & \multicolumn{2}{c}{ Eigenmannia tirlineata } & \multicolumn{2}{c}{ Eigenmannia virescens } \\
\hline GR (\%) & $\mathbf{N}$ & $\mathbf{( \% )}$ & $\mathbf{N}$ & $\mathbf{( \% )}$ & $\mathbf{N}$ & $\mathbf{( \% )}$ \\
\hline 0 & - & - & - & - & - & 11 \\
18,3 \\
25 & 7 & 11,7 & 32 & 53,3 & 19 & 31,7 \\
50 & 9 & 15 & 21 & 35 & 22 & 36,7 \\
75 & 16 & 26,6 & 7 & 11,7 & 8 & 13,3 \\
100 & 28 & 46,7 & - & - & 60 & 100 \\
\hline TOTAL & 60 & 100 & 60 & 100 & &
\end{tabular}

Índice de Importância Alimentar (IAi)

O item alimentar mais recorrente nas análises para as três espécies do gênero Eigenmannia foi inseto, seguido de material indefinido, este não sendo classificado como item, mas apresentando que o peixe ingeriu alimento e devido ao alto grau de decomposição do conteúdo presente no estômago não é possível à identificação, e por último e em menor expressão material vegetal também foi observado nas análises. A espécie E. macrops apresentou uma dieta bem diversificada explorando em maior quantidade insetos $(75,27 \%)$ composto principalmente por restos de larvas de Hymenoptera, pupas de Díptera, larvas de Trichoptera, material não identificado $(23,76 \%)$ e material vegetal $(1,04 \%)$ composto por pequenas folhas (Figura 3). Assim sendo, esta espécie foi classificada na guilda dos insetívoros.

A espécie $E$. trilineata explorou em maior quantidade insetos (62,70\%). As ordens de insetos mais encontrados para esta espécie foram: Díptera, Hemíptera, Coleoptera, larvas de Hymenoptera e 
Trichoptera, material não identificado correspondeu a (37,24\%) e material vegetal $(0,05 \%)$, sendo a porcentagem do item alimentar inseto abaixo de $70 \%$, pode assim ser classificada na guilda dos onívoro com tendência a insetívoria (Figura 4).

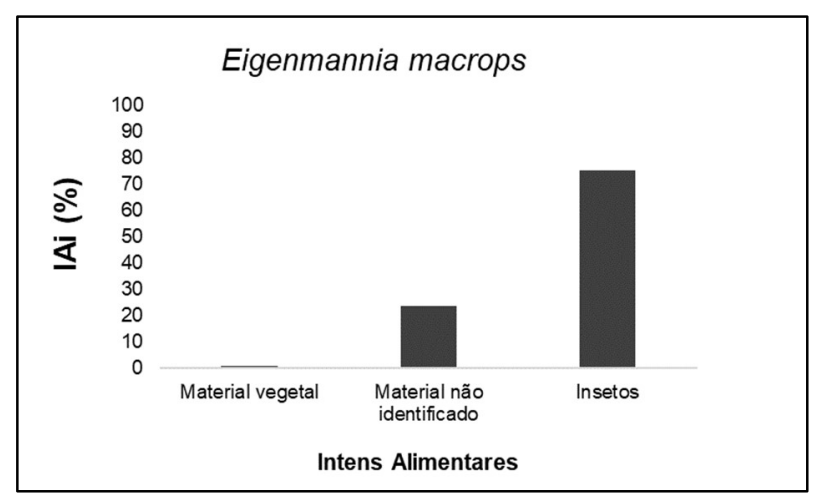

Figura 3: Índice de importância alimentar de Eigenmannia macrops.

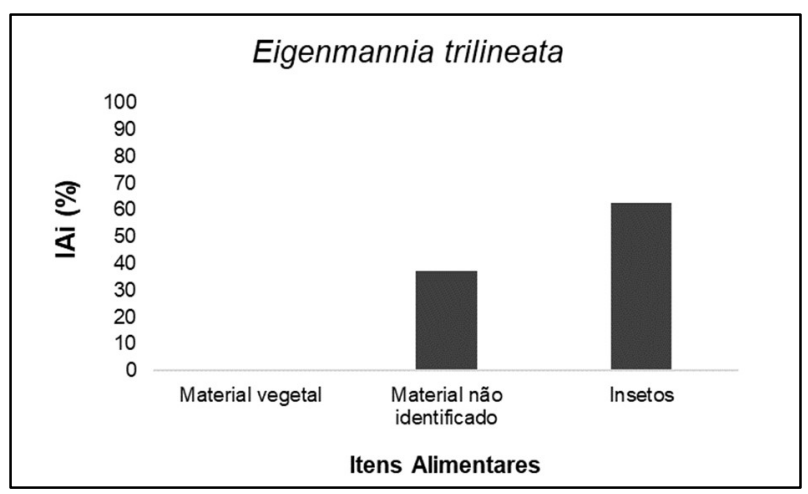

Figura 4: Índice de importância alimentar de Eigenmannia trilineata.

A espécie E. virescens explorou em maior quantidade insetos, apresentou índice alimentar (72,62\%), composto principalmente por restos de larvas de Hymenoptera, pupas de Díptera, Trichoptera, Hymenoptera, material não identificado correspondeu a (25,09\%) e material vegetal (2,28\%). Assim sendo, esta espécie foi classificada na guilda dos insetívoros (Figura 6).

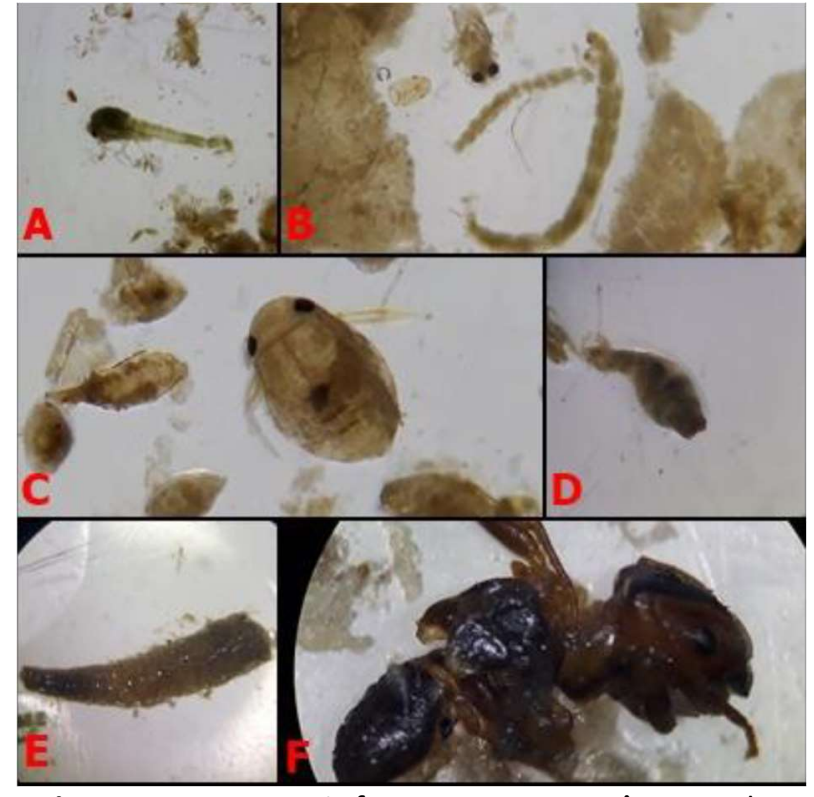

Figura 5: Insetos mais frequentes nos estômagos das espécies $E$. macrops, E. trilineata e E. virescens. A: pupa de Chironomidae (Díptera); B: Díptera e larvas de Hymenoptera C: Micronectidae (Hemíptera); D: Lepidóptera; E: Trichoptera; F: Hymenoptera.

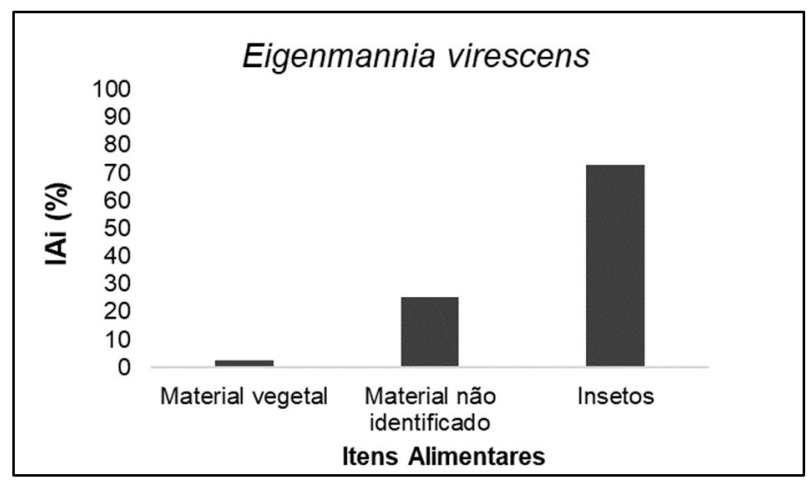

Figura 6: Índice de importância alimentar de Eigenmannia virescens.

Os insetos mais frequentes encontrados foram identificados a nível de Ordens: Hymenoptera (vespas), Hemíptera (percevejos), Lepidóptera (borboletas e mariposas), Coleóptera (besouros), Díptera (moscas) e Trichoptera (Figura 5). 


\section{Nicho trófico}

Os valores calculados para amplitude do nicho trófico através do índice padronizado de Levins foram de: E. trilineata 0,43170; E. macrops 0,3414; E. virescens 0,4308 (Figura 7). Quando o resultado deste índice está mais próximo de zero (0) há maior dissimilaridade quanto aos itens consumidos. Assim, os valores encontrados no presente estudo para E. trilineata e $E$, virescens de acordo com a escala de amplitude de nicho são considerados intermediárias $(0,4-0,6)$, já E. macrops teve a amplitude de nível baixa que sugere dieta restrita para espécie, podendo assim, ser considerada especialista, o que foi comprovado pelo índice de importância alimentar (IAi) com alto consumo de insetos em relação aos demais itens. Embora o valor da amplitude do nicho indique que a espécie $E$. virescens esteja classificada como uma espécie intermediária, o valor do IAi desta $(72,62 \%)$ indica que ela possui um hábito alimentar insetívoro. Talvez se houvesse um esforço de pesca maior e coletas nos outros períodos hidrológicos esse valor poderia se estabilizar para uma baixa amplitude nicho.

Dados de amplitude de nicho são bastante utilizados como complemento da composição da dieta dos peixes, sendo úteis, auxiliando na interpretação do grau de especialização alimentar de uma espécie.

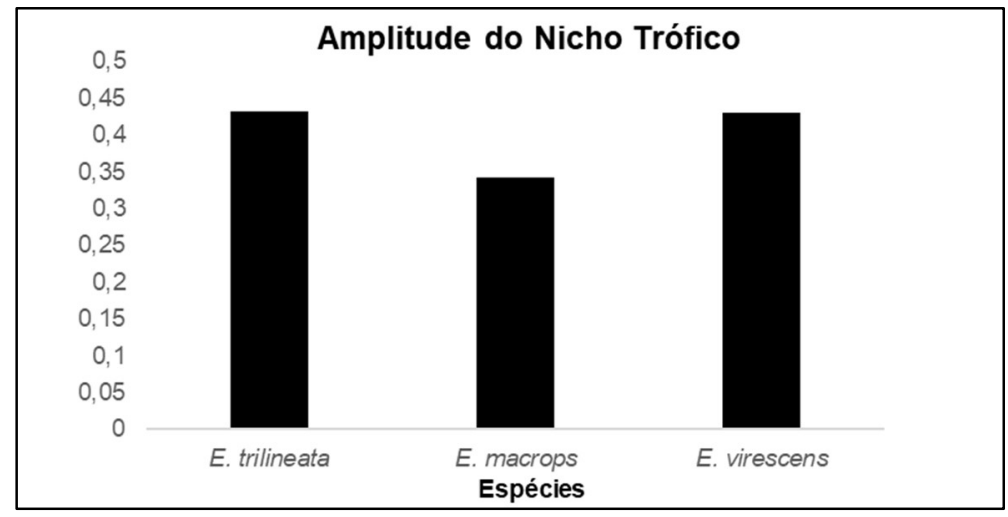

Figura 7: Amplitude de Nicho.

\section{Fator de condição}

Os valores médios obtidos para Fator de condição foram de: 1,899 da E. virescens, 0,108 da $E$. trilineata e 0.097 E. macrops (Figura 8). Estes demonstrando que a espécie E. virescens se encontrava em boas condições de bem-estar, já as demais apresentaram valores abaixo de 0 . Este resultado corrobora com o grau de repleção encontrado para a espécie E. virescens, na qual todos os estômagos analisados demonstraram ter algum item alimentar, assim confirmando o bem-estar do estado fisiológico da espécie. Já E. trilineata e E. macrops apresentando valores abaixo de 0 , assim podendo-se dizer que, mesmo não estando em boas condições fisiológicas, ambas ainda assim conseguiram explorar os recursos alimentares, como confirmado pelo grau de repleção. 


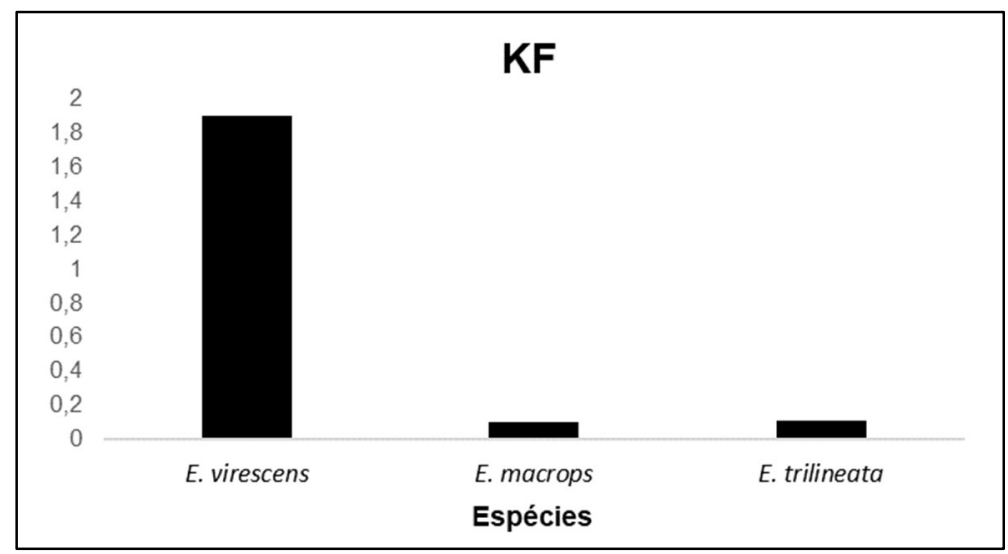

Figura 8: Gráfico de Fator de Condição de Fulton.

\section{DISCUSSÃO}

O resultado apresentado acima demonstra que as três espécies conseguem se alimentar e explorar os recursos disponíveis com frequência no ambiente de praia durante a seca, entretanto, em estudos decorrentes em lagos amazônicos (MÉRONA et al., 2004; MORTILLARO et al., 2015) retrataram que o período de seca é quando há uma escassez de alimentos para muitos peixes, especialmente os herbívoros e onívoros. Dentre os insetos identificados, as larvas de Hymenoptera e pupa de Chironomidae (Díptera) foram as mais frequentes em quase $100 \%$ dos estômagos analisados. Em análise de estrutura trófica de peixes em praias do baixo rio Negro (SILVA, 2017), Eigenmannia macrops e Eigenmannia virescens apresentaram categoria trófica como invertívoros autóctone. Na mesma área, em outro estudo para a verificação da alimentação de E. macrops, utilizando o apetrecho de pesca rede arrasto, Souza (2018) revelou que o principal item consumido foi inseto.

Pesquisando o hábito alimentar de Eigenmannia trilineata no parque estadual de Itapuã, Rio Grande do Sul (GIORA, 2004) obteve como resultados de itens alimentares consumidos em maior frequência microcrustáceos, seguido por larvas de Chironomidae, material indefinido, material vegetal e sedimento, validando com o que foi obtido nas análises do conteúdo para este trabalho. A espécie $E$. trilineata foi classificada na categoria de invertívoros bentônicos, por Claro Junior (2007), em estudo da dieta de peixes em ambientes de praias do rio Solimões-Amazonas, consumindo frequentemente os insetos: ninfas de Ephemeroptera, larvas de Megaloptera, Trichoptera, Hemíptera, além de larvas e pupas de Díptera, composta principalmente por Chironomaide.

A ocorrência de espécies generalistas ou especialistas em determinado ambiente é influenciada pela dinâmica dos recursos alimentares. Roughgarden (1974) relatou que os especialistas são melhores sucedidos que generalistas quando há o amplo suprimento de determinados recursos e estes são renováveis. Todavia, os peixes especialistas tornam-se vulneráveis quando estes recursos não são mantidos, podendo se tornar escassos, assim tendo a estratégia generalista como a mais vantajosa.

De acordo com (HAMADA et al., 2012), os insetos da ordem Hymenoptera possuem quatro asas membranosas, as posteriores menores que as anteriores acopladas por pequenos ganchos (hamuli). Possuem metamorfose completa, com as fases: ovo, larva, pupa e adulto (holometábolos). É uma ordem muito diversa, com importante papel ecológico, desde os polinizadores, dispersores de sementes aos que 
regulam a população de artrópodes. Seus representantes mais conhecidos são as vespas, formigas e abelhas. Os adultos são terrestres de vida livre.

Os insetos da ordem Hemíptera na fase adulta, geralmente, apresentam peças bucais picadorsugadoras, com origem na região anterior da cabeça e se estende até a parte ventral; ocelos presentes ou ausentes; antenas com a cinco artículos. Estas ordens contêm famílias aquáticas, semiaquáticas e terrestres. Algumas famílias são conhecidas como barata d'água, piolho d'água e percevejos patinadores. Ocorrem em diversos ambientes, tanto na superfície da água quanto submersos (PEREIRA, 2004; MERRIT et al., 2008; HAMADA et al., 2012; BUZZI, 2013).

Coleoptera apresenta insetos de pequeno a grande porte $(1$ até $15 \mathrm{~cm})$; tem peças bucais mastigadoras; hábitos alimentares diversificados; antenas variáveis quanto ao tamanho e forma; asas anteriores endurecidas (élitro). Apresentam metamorfose completa: ovo, larva, pupa e adulto (holometábolos). Podem ser aquáticos quando imaturos e adultos em algumas famílias e, em outras apenas um ou outro estágio é aquático. Estas podem ser encontradas em quase todo os biótopos (MERRIT et al. 2008; HAMADA et al., 2012).

A ordem Díptera apresenta alta diversidade com aproximadamente 24.000 espécies descritas para região Neotropical e, está distribuída em subordens como: Nematocera e Brachycera, que se diferenciam por tamanho de antenas, palpos maxilares e pelos estágios imaturos. Algumas famílias são conhecidas como moscas, mosquitos, pernilongos, borrachudos e mutucas. Os adultos são terrestres, as larvas podem ser terrestres ou aquáticas, podendo ter a cabeça bem desenvolvida ou não, tórax não possui três pares de pernas, podendo um par de pernas falsas estar presente. Representantes imaturos dessa ordem constituem grande parte da fauna de ecossistemas aquáticos como, rios, lagos, igarapés, poças temporárias, entre outros (HAMADA et al., 2012).

Os adultos de Trichoptera apresentam tamanho pequeno a médio; antenas longas e multiarticuladas; olhos compostos bem desenvolvidos; três ou nenhum ocelo; palpos bem desenvolvidos; dois pares de asas com cerdas, muitas veias longitudinais e poucas transversais; pernas desenvolvidas; as larvas possuem a cabeça e parte do tórax esclerosados. Na fase adulta podem ser encontrados próximos a lagos e cursos d'água, sob vegetação ou pedras. As larvas podem ser detritívoras, filtradoras, coletoras, cortadoras e predadoras, algumas são marinhas, sendo poucas terrestres ou ocasionalmente saem da água. Todas produzem seda para a construção de abrigos, casas e redes de captura de alimento no estágio larval. (WIGGINS, 2008; HAMADA et al., 2012).

As espécies do presente estudo após análises do conteúdo estomacal, demonstram que os itens que fazem parte de sua dieta são insetos e material vegetal, tendo os insetos em maior expressão, podendo assim, classificá-las como insetívoras, corroborando com (GIORA, 2004), em estudo para a espécie de E. trilineata, apresentando larvas de Chironomidae, material vegetal, material orgânico e insetos não identificados para indivíduos adultos.

Os valores encontrados no presente estudo sobre o nicho trófico para as espécies $E$. trilineata e $E$, virescens de acordo com a escala de amplitude de nicho são considerados intermediárias, já E. macrops 
teve a amplitude de nível baixa (CORRÊA et al., 2011), que sugere uma dieta restrita para espécie, podendo assim, ser considerada especialista (POUILLY et al., 2004), o que foi possível observar pelo índice de importância alimentar (IAi) com alto consumo de insetos em relação aos demais itens alimentares.

Pesquisando sobre o bem-estar de E. trilineata através o fator de condição (GIORA, 2004), afirmou que, entre machos e fêmeas esses valores não variam entre si, mas em relação ao que determina essa influência nos machos o peso dos estômagos foi determinante enquanto as fêmeas apresentaram o peso das gônadas.

\section{CONCLUSÕES}

Através das análises realizadas no presente estudo com as espécies $E$. macrops, E. trilineata e $E$. virescens, advindas de praias do rio Negro, a composição alimentar apresentou os itens insetos e material vegetal e, em ambas o item inseto demonstrou ser o principal recurso explorado por estas espécies, sendo, portanto, classificados na guilda dos insetívoros e onívoro com tendência a insetivoria.

A amplitude do nicho trófico corroborou coma informação do Índice de importância alimentar, apresentados valores próximos a zero, a espécie E. macrops foi descrita com seletiva. Os valores para $E$. trilineata e $E$. virescens resultaram padrão intermediário, porém com os dados obtidos no IAi, pode-se afirmar que a espécie $E$. virescens possui na sua dieta preferência pelo item alimentar inseto, sendo frequente o consumo.

O Fator de condição calculado paras as respectivas espécies apontou que os indivíduos da espécie Eigenmmania macrops se encontravam em boas condições de bem-estar, durante o período de seca, já $E$. trilineata e $E$. virescens mesmo apresentando valores abaixo de zero, demonstram que conseguem explorar os recursos alimentares.

\section{AGRADECIMENTOS}

Agradecimentos à Coordenação de Aperfeiçoamento de Pessoal de Nível Superior (CAPES), Fundação de Amparo à Pesquisa do Estado do Amazonas (FAPEAM) e Universidade Federal do Amazonas (UFAM).

\section{REFERÊNCIAS}

BUZZI, Z. J.. Entomologia didática. 6 ed. Curitiba: UFPR, 2013.

CORRÊA, C. E.; ALBRECHT, M. P.; HAHN, N. S.. Patterns of niche breadth and feeding overlap of the fish fauna in the seasonal Brazilian Pantanal, Cuiabá River basin. Neotropical Ichthyology, v.9, n.3, p.637-646, 2011. DOI: https://doi.org/10.1590/S1679-62252011000300017

CLARO JUNIOR, L. H.. Distribuição e estrutura tróficas das assembleias de peixes em praias do rio Solimões/Amazonas, Brasil. Tese (Doutorado) - Instituto Nacional de Pesquisas da Amazônia, Manaus, 2007. systematics, ecology and biogeography. In: LADICH, F.; COLLIN, S. P.; MOLLER, P.; KAPOOR, B. G.. Communication in Fishes. Enfield: Science Publishers Inc., 2006. p.647-696. DOI: https://doi.org/10.1016/i.jphysparis.2016.10.002

DAGOSTA, F. C. P.; PINNA, M. C. C.. The fishes of the Amazon: Distribution and biogeographical patterns, with a comprehensive list of species. Bulletin of the American Museum of Natural History, v.4, n.3, p.1-163, 2019. DOI: https://doi.org/10.1206/0003-0090.431.1.1

GOULDING, M.. História dos Rios Amazônicos. Brasília: Sociedade Civil Mamirauá, 1997.

GIORA, J.. Biologia reprodutiva e Hábito alimentar de Eigenmannia trilineata Lopéz \& Castello, 1996 (TELEOSTEI, 
STERNOPYGIDAE) do Parque Estadual de Itapuã, Rio Grande do Sul. Dissertação (Mestrado) - Universidade Federal do Rio Grande do Sul, Porto Alegre, 2004.

GIORA, J.; TARASCONI, H. M.; FIALHO, C. B.. Reproduction and feeding of the electric fish Brachyhypopomus gauderio (Gymnotiformes: Hypopomidae) and the discussion of a life history pattern for gymnotiforms from high latitudes. PLoS One, v.9, n.9, 2014. DOI:

https://doi.org/10.1371/journal.pone.0106515

HAMADA, N.; FERREIRA-KEPPLER, R. L.. Guia de insetos aquáticos e seminaquáticos da Reserva Florestal Ducke, Manaus, Amazonas, Brasil. Manaus: Universidade Federal do Amazonas, 2012.

HYSLOP, E. J.. Stomach contents analysis: a review of methods and their application. J. Fish Biol., v.17, p.411-429, 1980. DOI: https://doi.org/10.1111/j.10958649.1980.tb02775.x

INACIO, M. S.; ABELHA, M. C. F.. Estrutura trófica da assembleia de peixes de riacho Sul-Mato-Grossense. In: ENIC. Anais. 2016.

JUNK, W.; BAYLEY, P. B.; SPARKS, R. E.. The flood pulse concept in riverfloodplain systems. In: DOGDE, D. P.. Proceedings of the International Large River Symposium. Can. Spec. Publ. Fish. Aquat. Sci., 1989. DOI: https://doi.org/10.1201/b10749-2

KAWAKAMI, E.; VAZZOLER, G.. Método gráfico e estimativa de índice alimentar aplicado no estudo de alimentação de peixes. Bolm. Inst. Oceanogr., v.29, p.205-207, 1980. DOI: https://doi.org/10.1590/\$0373-552419800002000431980

LOWE-MCCONNELL, R. H.. Estudos ecológicos de comunidades de peixes tropicais. São Paulo: Edusp, 1999.

MÉRONA, B.; MÉRONA, J. R.. Food resource partitioning in a fish community of the central Amazon floodplain. Neotropical Ichthyology, v.2, n.2, p.75-84, 2004. DOI: https://doi.org/10.1590/S1679-62252004000200004

MÉRONA, B.; HUGUENY, B.; TEJERINA-GARRO, F. L.; GAUTHERET, E.. Diet-morphology relationship in a fish assemblage from a medium-sized river of French Guiana: the effect of species taxonomic proximity. Aquat Living Resour., v.21, p.171-184, 2008. DOI: https://doi.org/10.1051/alr:2008032

MILHOMEN, S. S. R.; CRAMPTON, W. G. R.; PIECZARKA, J. C.; SHETKA, G. H.; SILVA, D. S.; NAGAMACHI, C. Y.. Gymnotus capanema, a new species of electric knife fsh (GymnotidaeGymnotiformes) from eastern Amazonia, with comments on an unusual karyotype. Journal of Fish Biology, v.80, p.80215, 2012. DOI:

https://doi.org/https://doi.org/10.1111/j.10958649.2012.03219.x

MORTILLARO, J. M.; POUILLY, M.; WACH, M.; FREITAS, C. E. C.; ABRIL, G.; MEZIANE, T.. Trophic opportunism of central Amazon floodplain fish. Freshwater Biology, v.60, n.8, p.1659-1670, 2015. DOI: https://doi.org/10.1111/fwb.12598

PEREIRA, D. L. V.. Distribuição e chave taxonômica de gêneros de Gerromorpha e Nepomorpha (Insecta: Heteroptera) na Amazônia Central. Dissertação (Mestrado) - Universidade Federal do Amazonas, Manaus, Amazonas, 2004.

QUEIROZ, L. J. D.; TORRENTE-VILARA, G.; OHARA, W. M.; PIRES, T. H. D. S.; ZUANON, J.; DORIA, C. R. D. C.. Peixes do rio Madeira. São Paulo: Santo Antônio Energia, 2013.

REIS, R. E. J. S.; ALBERT, F.; DiDARIO, M. M.; MINCARONE, P.; PETRY, L. A.; ROCHA, L. A.. Fish biodiversity and conservation in South America. Journal of Fish Biology, v.89, n.1, p.12-47, 2016. DOI: https://doi.org/10.1111/jfb.13016

RESENDE, E. K.; PEREIRA, R. A. C.; SÓRIO, V. F.; GALVÃO, E. M.. Biologia da tuvira, Gymnotus cf. Carapo (Pisces, Gymnotidae) no baixo Rio Negro, Pantanal, Mato Grosso do Sul, Brasil. Embrapa Pantanal, 2006.

SILVA, T. M.. A resistência da teia alimentar antes e após a formação do reservatório da UHA Santo Antônio do Rio madeira. Dissertação (Mestrado em Desenvolvimento Regional e Meio Ambiente) - Universidade federal de Rondônia, Porto Velho, 2016.

SILVA, F. J.. Composição específica e a abundância da ictiofauna em ambientes de praias no baixo rio Negro, Amazonas, Brasil. Dissertação (Mestrado) - Universidade Federal do Amazonas, Manaus, 2017.

WIGGINS, G. B.. Larvae of the North American Caddisfly genera (Trichoptera). 2 ed. Toronto: University of Toronto Press Incorporated, 2008.

ZUANON, J.; FERREIRA, E.. Feeding ecology of fishes in the Brazilian Amazon: a naturalistic approach. In: CYRINO, J. E. P.; BUREAU, D. P.; KAPOOR, B. G.. Feeding and digestive functions of fishes. Enfield: Science Publishers, 2008. p.1-34. DOI: https://doi.org/10.1201/b10749-2

A CBPC - Companhia Brasileira de Produção Científica (CNPJ: 11.221.422/0001-03) detém os direitos materiais desta publicação. Os direitos referem-se à publicação do trabalho em qualquer parte do mundo, incluindo os direitos às renovações, expansões e disseminações da contribuição, bem como outros direitos subsidiários. Todos os trabalhos publicados eletronicamente poderão posteriormente ser publicados em coletâneas impressas sob coordenação da Sustenere Publishing, da Companhia Brasileira de Produção Científica e seus parceiros autorizados. Os (as) autores (as) preservam os direitos autorais, mas não têm permissão para a publicação da contribuição em outro meio, impresso ou digital, em português ou em tradução. 